\section{THE TREATMENT OF SEVERE CASES OF CHRONIC COLITIS.*}

By P. LOCKHART MUMMERY, B.C., F.R.C.S., SENIOR ASSISTANT SURGEON, ST. MARK'S HOSPITAL FOR DISEASES OF THE RECTUM AND TO THE QUEEN'S HOSPITAL, HACKNEY.

CASEs of chronic colitis naturally divide themselves into two distinct classes:

1. Those cases which if carefully treated by suitable dietary, proper regulation of the bowels, and perhaps one or more visits to some suitable spa where proper lavage of the bowel can be carried out, quickly get well, and remain well; and

2. Cases in which in spite of prolonged and careful medical and spa treatment, little if any improvement occurs, or the improvement is only temporary, the patient quickly relapsing and the symptoms in spite of continued treatment getting worse.

I think every one who has had any experience in the treatment of chronic colitis will admit that this second type of case not only exists but forms an unpleasantly large proportion of the whole.

The worst cases in this second class spend their time going from one doctor to another, and in visiting most of the English and Continental spas. Many of them go in for Christian Science, vegetarianism, or other forms of quackery. In fact, they form one of the worst classes of chronic invalids, a nuisance to their doctors, their relatives, and themselves. It naturally occurs to one to ask what is the reason that the treatment which seems so effectual in the first type fails so signally in the second. The reason is, I think, not far to seek-chronic colitis is a condition usually diagnosed from the symptoms alone, and I think we shall all admit that the treatment of a disease which is only manifest by its symptoms is always difficult, and owing to the large possibilities of error which must be present in any diagnosis founded only upon symptoms, often unsatisfactory.

During the last few years I have seen a considerable number of the more severe cases of chronic colitis, both in hospital and in private. Most of these cases had previously been treated medically for long periods, and many had also been to spas and had Plombières treatment. For the most part they belonged to the second class of cases that I have mentioned, and medical treatment had failed to give them any permanent relief. All the cases that I have seen have been examined with the sigmoidoscope, and in many an operation has been performed which has enabled me to examine directly the colon and so to ascertain the exact nature of the pathological lesion.

I have no hesitation in saying that all cases presenting symptoms of chronic colitis should be most carefully examined with a view of ascertaining the exact nature of the lesion in the colon. The chief reason for the unsatisfactory results that are often seen in cases of chronic colitis is that the symptoms ascribed to this disease may result from a great many different con ditions of widely different characters, and the treatment which is suitable for one case is not suited to others. Our aim in all such cases should be to find out if possible the real cause of the symptoms. This is admittedly not always possible, but, on the other hand, it often is.

In a paper read before the Royal Medical and Chirurgical Society in June, 1907, I first pointed out that in the great majority of cases of chronic colitis a lesion or lesions could by suitable means be demonstrated in the colon, that these lesions varied considerably in different cases, and that any treatment which did not take into account the cause of the symptoms is unsound in principle.

The name "chronic colitis" should be reserved for those cases in which there is a chronic inflammatory condition of the mucous membrane of the colon. They can be distinguished readily by examination with the sigmoidoscope, as the sigmoid flexure is practically always involved.

The cases in which there is a definite inflammatory condition of the mucosa, as seen on sigmoidoscopic examination, are those which are suitable for treatment by lavage and the Plombières douche. Many of them in my experience do well. They form the first class of which I have spoken, and the majority of all cases. On the other hand, there are many cases with identical symptoms in which, on examination with the sigmoidoscope, we find the mucous membrane quite normal, but some other lesion is present. It is this class of case which I propose to consider in detail. In many there is no inflammation of the mucosa of the bowel, while in others in which there is, it is purely secondary to some other lesion. The nature of the lesion varies considerably. An important class is that in which some chronic obstructive lesion exists in the colon, such as adhesions from previous peritonitis or perimetritis. Or a kink or acute angle is present in the pelvic colon as the result of adhesions to the iliac fossa or in the meso-sigmoid, probably due to old chronic constipation. Another form of lesion is that in which there is a partial volvulus of the sigmoid, or a chronic and recurring intussusception.

We must also bear in mind that cancer of the colon may cause symptoms of chronic colitis, which are indis tinguishable from those occurring in the simpler forms. I have seen seven such cases, in several of which the patient had previously undergone treatment for months for a supposed simple chronic colitis.

In another well-marked group of cases we find a chronically inflamed appendix, which, owing to the fact that it has not caused the usual distinctive symptoms, has remained undetected. There is no doubt that a chronically inflamed appendix may cause symptoms of chronic colitis, though exactly how it does so is not always clear. In some instances it is due to adhesions which have formed to the pelvic colon, while in others it probably produces symptoms by the constant discharge of septic material into the bowel, in the same way that carious and septic teeth will cause gastric disease.

There is one well-marked group of cases in which all the symptoms of a severe chronic mucous colitis exist, and in which there is no inflammation of the mucosa, but the whole colon is found to be lax and atonic. On examination with the sigmoidoscope the bowel is seen to be unduly lax and to have a marked tendency for the upper portions to prolapse into the lower; the walls are thin and semitransparent; the lumen is dilated, and the normal sacculation much exaggerated. There is generally well-marked ptosis of the transverse colon and of the stomach. In several instances in which I have operated for this condition the centre of the transverse colon has been found in the bottom of the pelvis lying in front of the other part of the rectum. In this type of case the colon is dilated and its walls atonic, but there is no inflammation of the mucosa. It is obvious that it is most important to be able to distinguish this type of case, as treatment by lavage which involves distension of the already dilated colon will not only be useless but will do harm. Such cases are not suitable for Plombières treatment. It is quite obvious that these different conditions, although they cause the same symptoms, cannot be classed as one disease.

I cannot believe that the neurotic element which is so commonly present, and about which we hear so much, is other than secondary. I have frequently seen it entirely disappear after the cause of the symptoms has been removed.

One of the chief reasons for the unsatisfactory results that have been obtained in the treatment of many cases of colitis has been inaccurate diagnosis, and treating as a disease what is only a symptom.

The indications for operating in cases of chronic colitis will, of course, depend upon the nature of the lesion present. When there is any evidence of an obstructive lesion such as adhesions or chronic volvulus, laparotomy is clearly indicated, and affords the only possible method of adequately dealing with the case.

The following is a good instance of the type of case which can only be treated satisfactorily by operation.

The patient, a married lady, was recently sent to me by her doctor. For ten years she had been a chronic invalid with mucous colitis. She suffered from chronic pain in the abdomen which at times became severe, and was always worst on the left side. She had lost weight, and always felt ill and depressed. She had fits of weeping, and misery on the slightest, and of ten upon no, provocation, and was unable to go about or enjoy upon no, provocation, and was unable to go about or enjoy life in the ordinary way. She had an earthy complexion, and her appearance when I saw her was typical of toxaemia or auto intoxication. The stools contained large quantities of mucus, and of ten consisted of little else. A curious and unusual sym ptom was that the presence of anything in the rectum caused 
an uncontrollable desire to go to stool and much tenesmus. She had been under medical treatment for years, and all the recognized forms had been tried. On examining the bowel with the sigmoidoscope I found the mucosa quite normal in appearance. In the middle of the sigmoid, how ever, the bowel was found to be firmly fixed and angulated, apparently by adhesions. The uterus was also found to be markedly retroflexed. It seemed probable that the tenesmus from which she suffered was due to the condition of the uterus, and a gynaecologist who saw her with me confirmed this view.

I opened the abdomen and found a number of firm adhesions binding down and kinking the middle of the sigmoid flexure; these were divided and the wound left in the peritoneum sewn up. The uterus was also drawn forward and anchored to the abdominal wall go as to correct the position.

The patient made a good recovery, and all her symptoms have now completely disappeared. When I last saw her some months after the operation she had put on weight, her complexion was me that she never $r \in$ membered feeling so well and fit.

Though most of the cases of true colitis (that is to say, when a chronic inflammation of the mucosa of the colon is found) get well as the result of medical treatment and lavage, there are nevertheless a certain number which either fail to get entirely well or suffer from frequent relapses. These cases are, I believe, best treated by appendicostomy, which enables the patient to keep the colon efficiently washed out daily with the minimum of inconvenience, and the symptoms quickly clear up.

The most difficult cases to treat are perhaps those in which there is a general atony of the bowel combined with ptosis of the colon. Stitching up the colon is quite useless, as it is unreasonable to expect any stitches to hold up the stomach and transverse colon permanently when the natural supports have not been sufficient to do so. It is impossible to deal directly with the condition, but I have had very satisfactory results in such cases from appendicostomy, and the fitting of a belt to support the abdomen. The object of the operation in such cases is to prevent the stagnation of the faecal contents of the colon, which is the chief cause of trouble, and at the same time by the introduction of suitable peristaltic stimulants to improve and restore the muscular tone of the bowel wall.

The following well illustrates this type of case :

Mrs. B., aged 32, a married lady, was sent to me by her doctor suffering from severe chronic colitis. There was a history dating back twelve sears, though the symptoms had only been really severe for the last four years. She always felt ill, and had a chronic aching pain in the left side of the abdomen. She was always very constipated, and had to take large dóses of aperients, and this often without success. The stools contained mucus, and of ten large casts over a foot in length. She had been treated for long periods by the usual remedies, and had on different occasions consulted several well known physicians, but without obtaining any relief from her symptoms.

Framination showed an atonic condition of the bowel, and chronic inflammatory changes of the mucosa. There was also well-marked ptosis of the transverse colon.

I operated, and on opening the abdomen found the colon much dilated and thin. There was also considerable enteroptosis. Appendicostomy was performed, as the only alternative seemed to be removal of the colon.

The colon was subsequently irrigated daily with warm water (at first in small quantities) to which a teaspoonful of ox bile was added. She rapidly improved in health, and learnt to wash out the bowel for herself. The mucus entirely disappeared from the stools, her health improved, and the bowels acted without any aperient being used. During the next six months she put on 2 st. in weight, and when I last heard from her, about nine months after the operation, she was quite well, and although on my advice still washing out the bowel, was quite freefrom any of her old symptoms.

Appendicostomy is an operation followed by excellent results in many of these cases, but it is not a panacea for all cases of so-called chronic colitis any more than is lavage. It should only be performed in suitable cases, and that can only be done if we first ascertain the cause of the symptoms: I have seen cases in which appendicostomy has failed even to relieve the symptoms because there was some obstruction or other lesion which had not been detected.

I do not wish it to be thought that I am advocating operation in all cases in which there are symptoms of chronic colitir. I think operation should only be resorted to after a careful trial has been given to medical treatment. But the important thing is to ascertain the cause of the symptoms by a careful examination aided whenever possible by the use of the sigmoidoscope, as it is useless treating a case by medical measures where there is an obstructive lesion in the colon, or a chronically inflamed and thickened appendix. And, in any ease, it is not satisfactory to institute treatment without knowing the cause of the symptoms.

ENTEROSPASM : OPERATION : DEATH. By VAUGHAN PENDRED, M.D.DURH., F.R.C.S.ENG. COVENTRY.

Mr. C. W. Dran's case of enterospasm reported in the JourNaL of March 13th recalls a similar case I had under my care four years ago, which ended fatally, and on which I obtained a partial autopsy:

The patient was a querulous, excitable, grey-haired little woman aged 57 when I first saw her in October, 1904. She was fat and healthy-looking. For the preceding three years she had suffered from time to time from colic with vomiting and diarrhoea. Latterly these attacks had become very frequent and severe. The tongue was coated. The urine showed a trace of albumen, and she complained of frequent micturition. During the next two months, in spite of energetic treatment, she had much colic and some emesis, and had plainly emaciated. A copious bleeding from the rectum occurred about this time.

In January, 1905, I sent her to Guy's Hospital, where some improvement took place during her month's residence. An $x$-ray photograph of the abdomen was negative. Movable right kidney was diagnosed and the wearing of a belt advised.

Month after month she continued to waste, and had constant vomiting attacks. Constipation alternated with diarrhoea, which latter somewhat relieved her pain.

In July, 1905 - or ten months after my first notes-she was nearly bedridden with colic, coming on every few minutes, accompanied by tremendous borborygmi. Visible peristaltic waves passed across coils of intestine from left to right every few minutes, as though the intestine were endeavouring to overcome some obstruction. By the end of this month her condition was pitiable, and I had to keep her constantly under the influence of morphine. The pain was almost constant night and day. The vomit now became stercoraceous, hiccough supervened, and the bowels were confined. In short, the symptoms pointed to intestinal obstruction.

On July 29 th I opened the abdomen below the umbilicus, but at first could find nothing amiss. Some feet of small intestine, distended but healthy looking, prolapsed through the wound. At one point the distension of the gut suddenly ceased, and the distal portion was flat, toneless, and of a paler colour, so that I thought that I had discovered the obstruction that I sought. Whilst I was considering what I would do next, the collapsed gut began to fill out again just as it had appeared to do through the abdominal wall. In order to return the gut it was necessary to incise a prominent coil, from which gas and foul liquid faeces escaped.

Three days later her condition was as bad as ever. The gurgling, peristalsis, pain and sickness, with occasional haematemesis, being nearly continuous. She died in the middle of September, emaciated to a skeleton, as she was unable to take food for many days. The peristaltic waves were a marked symptom up to the last moment of her life.

A post-mortem examination of the abdomen was all I could obtain leave to make. Every organ, though wasted, was macroscopically healthy. The intestine was partly opened up, and presented a normal appearance. Portions of the organs were sent to a London pathologist, who confirmed my opinion of them.

This case parallels Mr. Dean's in many particulars. Both patients had suffered from abdominal pains, vomiting, and bowel trouble for some years. The appearance of the ileum at the operation was similar. In Dr. Ashe's and my case the flattened gut "livened up" under observation. Anorexia and utterly uncontrollable vomiting, with occasional haematemesis and melaena, were such prominent symptoms in my case, that they point to a profound toxaemia, and not merely to a disturbance of innervation of the intestine.

\section{FISTULAE BETWEEN THE STOMACH AND BILE PASSAGes, ETC.}

By ALEXANDER DON, C.M., F.R C.S., M.A., SURGEON, ROYAL INFIRMARY, DUNDEE.

The cases of unusual fistulae in the Jodrnas of May lst by Mr. Mayo Robson make one think that the unrecorded cases may be more numerous than would be expected, and the following three may be of some interest:

M. L., aged 32, a multipara, whom I saw with Dr. Lowson on June 17tb, 1899, had the following history: From November 1898, she had repeated attacks of biliary colic, which developed in intensity till April 5th, 1899. The vomit was at times 